\title{
Characterizing sensitivity and coverage of clinical WGS as a diagnostic test for genetic disorders
}

\author{
Yan Sun ${ }^{1 \dagger}$, Fengxia Liu ${ }^{2,3+}$, Chunna Fan ${ }^{2,3 \dagger}$, Yaoshen Wang ${ }^{2,3 \dagger}$, Lijie Song ${ }^{2,3}$, Zhonghai Fang ${ }^{2,3}$, Rui Han ${ }^{2,3}$, \\ Zhonghua Wang ${ }^{2,3}$, Xiaodan Wang ${ }^{2,3}$, Ziying Yang ${ }^{2,3}$, Zhenpeng X ${ }^{1}$, Jiguang Peng ${ }^{1}$, Chaonan Shi ${ }^{2,3}$, \\ Hongyun Zhang ${ }^{1}$, Wei Dong ${ }^{4}$, Hui Huang ${ }^{1}$, Yun Li ${ }^{1}$, Yanqun Le ${ }^{2}$, Jun Sun ${ }^{2,3^{*}}$ and Zhiyu Peng ${ }^{1 *}$ (0)
}

\begin{abstract}
Background: Due to its reduced cost and incomparable advantages, WGS is likely to lead to changes in clinical diagnosis of rare and undiagnosed diseases. However, the sensitivity and breadth of coverage of clinical WGS as a diagnostic test for genetic disorders has not been fully evaluated.

Methods: Here, the performance of WGS in NA12878, the YH cell line, and the Chinese trios were measured by assessing their sensitivity, PPV, depth and breadth of coverage using MGISEQ-2000. We also compared the performance of WES and WGS using NA12878. The sensitivity and PPV were tested using the family-based trio design for the Chinese trios. We further developed a systematic WGS pipeline for the analysis of 8 clinical cases.

Results: In general, the sensitivity and PPV for SNV/indel detection increased with mean depth and reached a plateau at an $\sim 40 \mathrm{X}$ mean depth using down-sampling samples of NA12878. With a mean depth of 40X, the sensitivity of homozygous and heterozygous SNPs of NA12878 was $>99.25 \%$ and $>99.50 \%$, respectively, and the PPV was 99.97\% and $98.96 \%$. Homozygous and heterozygous indels showed lower sensitivity and PPV. The sensitivity and PPV were still not 100\% even with a mean depth of $150 X$. We also observed a substantial variation in the sensitivity of CNV detection across different tools, especially in CNVs with a size less than $1 \mathrm{~kb}$. In general, the breadth of coverage for disease-associated genes and CNVs increased with mean depth. The sensitivity and coverage of WGS ( 40X) was better than WES $(\sim 120 \mathrm{X})$. Among the Chinese trios with an $\sim 40 \mathrm{X}$ mean depth, the sensitivity among offspring was $>99.48 \%$ and $>96.36 \%$ for SNP and indel detection, and the PPVs were $99.86 \%$ and $97.93 \%$. All 12 previously validated variants in the 8 clinical cases were successfully detected using our WGS pipeline.

Conclusions: The current standard of a mean depth of 40X may be sufficient for SNV/indel detection and identification of most CNVs. It would be advisable for clinical scientists to determine the range of sensitivity and PPV for different classes of variants for a particular WGS pipeline, which would be useful when interpreting and delivering clinical reports.
\end{abstract}

\footnotetext{
*Correspondence: sunjun@bgi.com; pengzhiyu@bgi.com

${ }^{\dagger}$ Yan Sun, Fengxia Liu, Chunna Fan and Yaoshen Wang contributed

equally to this work

1 BGI Genomics, BGI-Shenzhen, Shenzhen 518083, China

${ }^{2}$ Tianjin Medical Laboratory, BGI-Tianjin, BGI-Shenzhen, Tianjin 300308,

China

Full list of author information is available at the end of the article
}

(c) The Author(s) 2021. Open Access This article is licensed under a Creative Commons Attribution 4.0 International License, which permits use, sharing, adaptation, distribution and reproduction in any medium or format, as long as you give appropriate credit to the original author(s) and the source, provide a link to the Creative Commons licence, and indicate if changes were made. The images or other third party material in this article are included in the article's Creative Commons licence, unless indicated otherwise in a credit line to the material. If material is not included in the article's Creative Commons licence and your intended use is not permitted by statutory regulation or exceeds the permitted use, you will need to obtain permission directly from the copyright holder. To view a copy of this licence, visit http://creativecommons.org/licenses/by/4.0/. The Creative Commons Public Domain Dedication waiver (http://creativeco mmons.org/publicdomain/zero/1.0/) applies to the data made available in this article, unless otherwise stated in a credit line to the data. 
Keywords: WGS, Sensitivity and PPV, DP and breadth of coverage, CNV, Clinical diagnosis

\section{Background}

Recently, whole-exome sequencing (WES) and whole genome sequencing (WGS) have been routinely used and are gradually being optimized for the detection of rare and common genetic variants in humans [1-4]. Several studies have compared the performance of WES and WGS in both technical aspects and clinical aspects [5, 6]. For example, focusing on mappability, Barbitoff et al. assessed the coverage of coding regions provided by several modern WES platforms and WGS [5]. Scala et al. compared the diagnostic performance of WES and WGS in children and adults with epilepsy [6]. In comparison to WES, WGS is more powerful for detecting variants. Theoretically, WGS has the potential to identify nearly all forms of genetic variation [7], including single-nucleotide variants (SNVs), in both the protein-coding and noncoding regions (such as introns and promoters) of the genome, small insertions/deletions (indels), and copynumber variants (CNVs) $[8,9]$. Without target region selection, WGS could provide a more uniform DP for the genome, making the detection of CNVs easier. Moreover, WGS could provide higher sensitivity and a higher yield in variant detection in the coding regions [10-12]. Several studies have demonstrated the advantages of WGS for variant detection [13-16]. For patients with highly suspected genetic disorder, WGS might be optimal for further evaluation when the patient remains undiagnosed after clinical WES. With the reduction of the cost of sequencing and its incomparable advantages, WGS is likely to change the clinical diagnosis of rare and undiagnosed diseases and is bound to become a routine part of clinical care in the near future.

The analysis of a clinical WGS usually starts from quality evaluation. Mean DP is often recognized as a general indicator of overall sensitivity. It has been reported that variant calling is more reliable with increasing DP [17]. As a crucial factor in data quality, most centers conducting MPS technology would determine thresholds for average DP across WES/WGS and determine the minimum DP that must be achieved for a certain fraction of target bases [18]. The breadth of coverage describes the fraction of the total target genomic region that is sequenced to an adequate depth in a particular assay [4]. The American College of Medical Genetics and Genomics (ACMG) recommends that 90\%-95\% breadth of coverage above a minimum threshold of $10 \mathrm{X}$ should be achieved for exome data with an average depth of 100X [18]. Thus far, a mean depth of 30-50X is the most widely used mean DP for WGS $[19,20]$. However, the sensitivity and breadth of coverage of clinical WGS have not been fully evaluated, especially for $\mathrm{CNV}$ detection, some of which are associated with human disease [21-24]. For clinical WGS, the sensitivity and coverage of CNVs have not been comprehensively investigated.

In this study, we performed a systematic analysis of the sensitivity and coverage of clinical WGS using 5 gold standard samples (NA12878, YH, NA24631, NA24694 and NA24695). Then, we applied clinical WGS for the reanalysis of 8 clinical cases with known disease-causing variants. The results may provide a reference for laboratories that perform clinical WGS.

\section{Methods}

\section{Sequencing of samples}

Based on the study design, DNA samples of NA12878, NA24631, NA24694 and NA24695 were procured from Coriell (Camden, NJ). One microgram of DNA was used for $\mathrm{YH}$ and the GIAB Chinese family to generate pairedend reads of $100 \mathrm{bp}$. The most widely used NA12878 was sequenced 2 times (WES and WGS) (Table 1). This study and all the protocols followed herein were approved by the ethics committee of BGI (NO. BGI-IRB19143).

\section{Alignment and variant calling}

In this study, a standard bioinformatics pipeline was used for analysis of all the samples (Additional files 1,

Table 1 Sample information

\begin{tabular}{lllllll}
\hline Sample name & Sequencing & Platform & DNA input & PCR/PCR-free & Read length & Mean depth \\
\hline NA12878-1 & WGS & MGISEQ-2000 & $1 \mu \mathrm{g}$ & PCR & PE100 & 197 \\
NA12878-2 & WES & MGISEQ-2000 & $300 \mathrm{ng}$ & PCR & PE100 & 220 \\
YH & WGS & MGISEQ-2000 & $1 \mu \mathrm{g}$ & PCR & PE100 & 151 \\
NA24631 & WGS & MGISEQ-2000 & $1 \mu \mathrm{g}$ & PCR & PE100 & 111 \\
NA24694 & WGS & MGISEQ-2000 & $1 \mu \mathrm{g}$ & PCR & PE100 & 115 \\
NA24695 & WGS & MGISEQ-2000 & $1 \mu \mathrm{g}$ & PCR & PE100 & 117 \\
\hline
\end{tabular}


2). To eliminate variability due to differences among various bioinformatics tools, we developed a standard bioinformatics pipeline, which included current widely used tools (Additional files 1, 2). In general, after removal of sequencing adapters and low-quality reads, "clean reads" were aligned to the GRCh37 with BWA 0.7.12-r1039 [25]. Genome Analysis Toolkit (GATK)package-4.0.11.0 [26] MarkDuplicates was used to remove duplicate reads. After realignment around indels and quality score re-calibration using GATKpackage-4.0.11.0 [27], VCF files were generated using GATK for further analysis. For the CNV detection, 3 widely used tools (CNVnator [28], BreakDancer [29] and LUMPY [30]) were performed in this study.

After trimming sequencing adapters and consecutive low-quality bases using fastp [31], the clean reads of NA12878-1 were down-sampled by the sequencing depth of 10X (NA12878-1_10X), 20X (NA12878-1_20X), 30X (NA12878-1_30X), 40X (NA12878-1_40X), 50X (NA12878-1_50X), 60X NA12878-1_60X), 70X (NA12878-1_70X), 80X (NA12878-1_80X), 90X (NA12878-1_90X), 100X (NA12878-1_100X), 120X (NA12878-1_120X) and 150X (NA12878-1_150X) using seqtk (https://github. com/lh3/seqtk). The high-coverage NA12878-1 sample was sequenced with the MGISEQ-2000 platform to an average depth of $\sim 197 \mathrm{X}$ (PE100) using PCR-based library construction. The clean reads of $\mathrm{YH}$ were also down-sampled by the sequencing depth.

\section{Sensitivity and positive predictive value of variant calls}

To evaluate the performance of variant calls in detecting true genotypes, the high-confidence calls (SNPs and indels) for GIAB sample HG001 (NA12878) and HG005/HG006/HG007 (Chinese son/father/mother) were considered true-positive calls (v3.3.2) [32]. We restricted the calculation of sensitivity (high-confidence calls detected by our method/all high-confidence calls in GIAB), specificity (sites called as a reference using our method/all reference sites in GIAB), accuracy (percent of calling agreement of our method when compared with GIAB) and PPV (high-confidence calls detected by our method/all variants detected by our method) of variant calls to the high confidence region (v3.3.2) [32]. High-confidence variant calls and regions tend to include a subset of variants and regions that are easier to detect [32]. The sensitivity and PPV of variant calls in the $\mathrm{YH}$ sample were also evaluated. The alleles validated by the Illumina $1 \mathrm{M}$ BeadChip were considered "true-positive" calls for YH [33]. Genotype quality (GQ) and DP were used to filter out variants with erroneous variant calls.

\section{Breadth of coverage for disease-associated genes and CNVs}

To assess the recommended depth for proband-only WGS in clinical diagnostics, we collected a total of 6 gene sets for coverage analysis of disease-associated genes: ACMG59 [34], ClinVar [35] (3824 genes, accessed on 19 February 2019), Genetic Home Reference [36] (1471 genes, accessed on 2 July 2019), HGMD [37] (8171 genes, professional March 2018), OMIM [38] (3835 genes, accessed on 4 April 2018) and Orphanet [39] (2405 genes, accessed on 2 July 2019). For the annotation of gene regions, we used the information available in NCBI annotation release 104. For different transcripts, we first used the transcripts used in the HGMD database. The rest of the gene region consisted of a combination of the regions of all transcripts. Coverage analysis of the 12 down-sampling samples of NA12878-1 and YH for the 6 gene sets was performed for evaluation.

The genes in a single gene set of the 6 gene sets were incomplete. To generalize a new gene list containing all the putative disease-associated genes, we compiled a list of 8394 putative disease-associated genes from the 6 gene sets (Additional file 2: Table S9). This new gene set was generated using the following criteria: (1) a gene was retained when it was recorded with an association status of "assessed" in the Orphanet database and had one of the following association types: (a) "Disease-causing germline mutation(s) in", (b) "Disease-causing germline mutation(s) (gain of function) in", (c) "Disease-causing germline mutation(s) (loss of function) in", (d) "Major susceptibility factor in", (e) "Modifying germline mutation in", (f) "Role in the phenotype of", (g) "Candidate gene tested in"; (2) a gene was retained when it was recorded as a disease-related gene in the Genetic Home Reference database; (3) a gene was retained when it was recorded as a disease-related gene and the molecular basis of the disease was known, unless the inheritance of the disease was recorded as "SMu" (somatic mutation) only in the OMIM database; (4) a gene was retained when at least one variant of the gene was recorded as a probable/possible pathological mutation in the HGMD database; (5) a gene was retained when at least one variant of the gene was recorded as pathogenic or likely pathogenic in the ClinVar database; (6) a gene was retained unless it was not recorded with a well-defined gene ID or genome coordinate information in NCBI annotation release 104. This new gene list was used in the comparison of WES and WGS and is ideal for coverage analysis of clinical WGS.

We also performed coverage analysis of the 12 downsampling samples of NA12878-1 and $\mathrm{YH}$ for CNVs described in the DECIPHER database (GRCh37_v9.29). Detailed information can be found in Additional file 2: Table S4. 


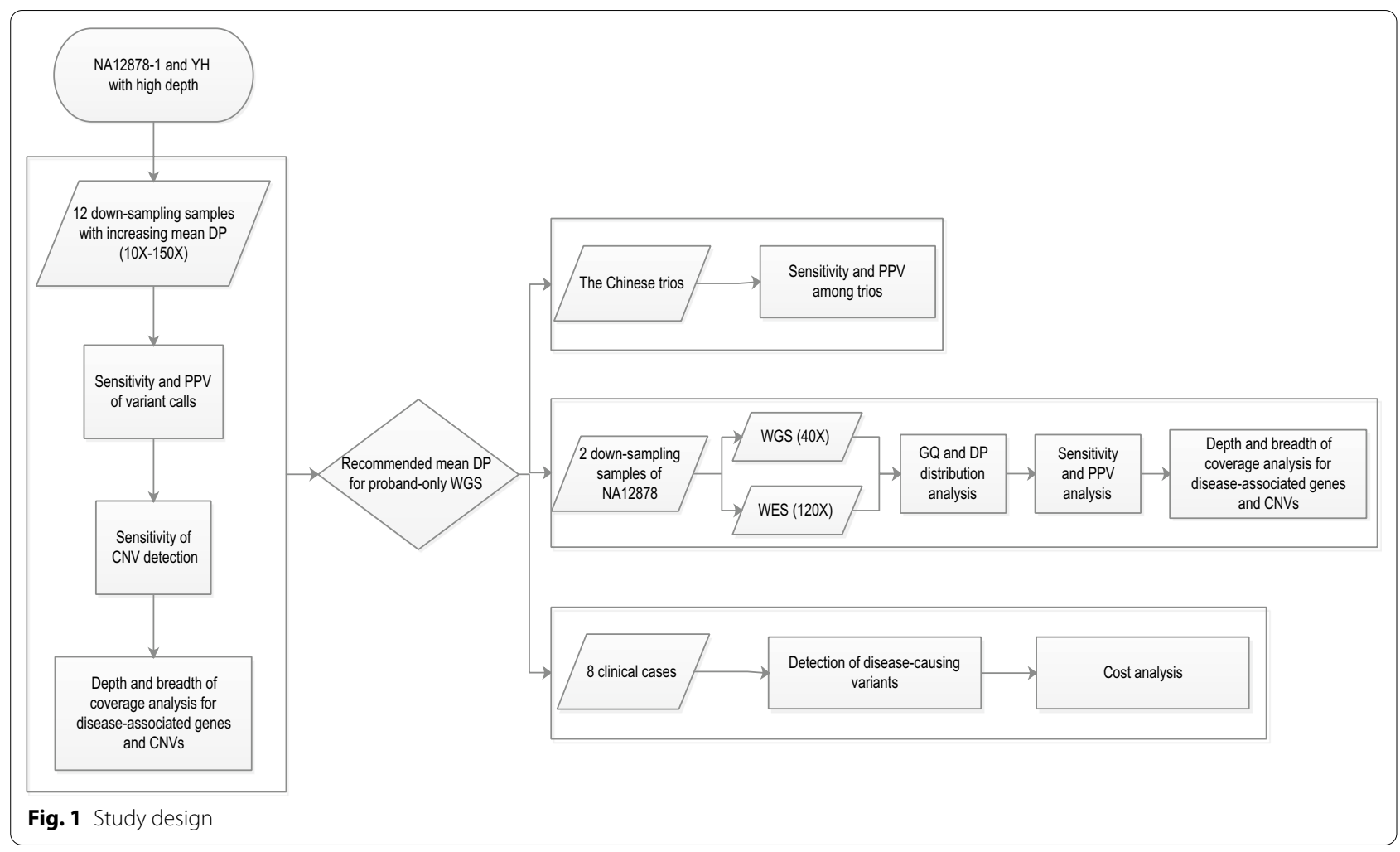

\section{CNV analysis}

Unlike SNPs and indels, there is no perfect "gold standard" CNV dataset for benchmarking. In this study, to assess the recommended depth for proband-only WGS in clinical diagnostics, we evaluated the sensitivity of CNV detection using 3 CNV call sets of NA12878 from published papers [40-42]. CNV call set 1 was also used by Haraksingh et al. [40] for the benchmarking of CNV detection from 17 commercially available arrays and low-coverage WGS. CNV call set 2 was determined by a machine learning-based approach (svclassify) and obtained from the GIAB Consortium benchmark SV calls resource [41]. CNV call set 3 included 874 deletions detected by both reference-based (a custom pipeline and PBHoney using both raw and error-corrected reads) and assembly-based analysis via single-molecule technologies [42]. All 3 CNV call sets have been previously compiled from NA12878 for benchmarking and downloaded for further analysis in the current study (Additional file 2: Table S10).

In the 12 down-sampling samples of NA12878-1, CNVnator (v0.3.2) [28], BreakDancer (v1.4.5) [29] and LUMPY (v0.2.13) [30] were assessed for CNV detection sensitivity with default or recommended parameters. In a $\mathrm{CNV}$ call set, true positives were classified as CNVs with at least a 50\% reciprocal overlap with CNVs in the call set (BEDTools) [43]. For benchmarking, we determined the number of gold standard CNVs detected in the 12 downsampling samples of NA12878-1 for the $3 \mathrm{CNV}$ call sets.

\section{Sensitivity and PPV of variant calls in the Chinese trios}

To test the sensitivity and PPV of variant calls for triobased WGS, we investigated the sensitivity and PPV when taking advantage of the family-based trio information in the Chinese trios. Using the segregation pattern, we focused on the autosomes and X chromosome of NA24631, NA24694 and NA24695. Taking advantage of the family-based trio design, we analyzed all variants (DP $\geq 10 \mathrm{X}$ ) of both parents, where one parent was consistently called as homozygous for the reference allele and the other as homozygous for the alt allele. We then used the variant calls (SNPs and indels) in the offspring to test the sensitivity and PPV for these loci.

\section{Results \\ Study design}

To test the sensitivity and coverage of clinical WGS, the Genome in a Bottle (GIAB) sample HG001 (NA12878), HG005 (NA24631)/HG006 (NA24694)/HG007 (NA24695) (known as the Chinese son/father/mother) and $\mathrm{YH}$ cell line (a human lymphoblastoid cell line from first Asian genome donor) [33] were collected and sequenced using the MGISEQ-2000 platform. All the samples used in this study are listed in Table 1. 


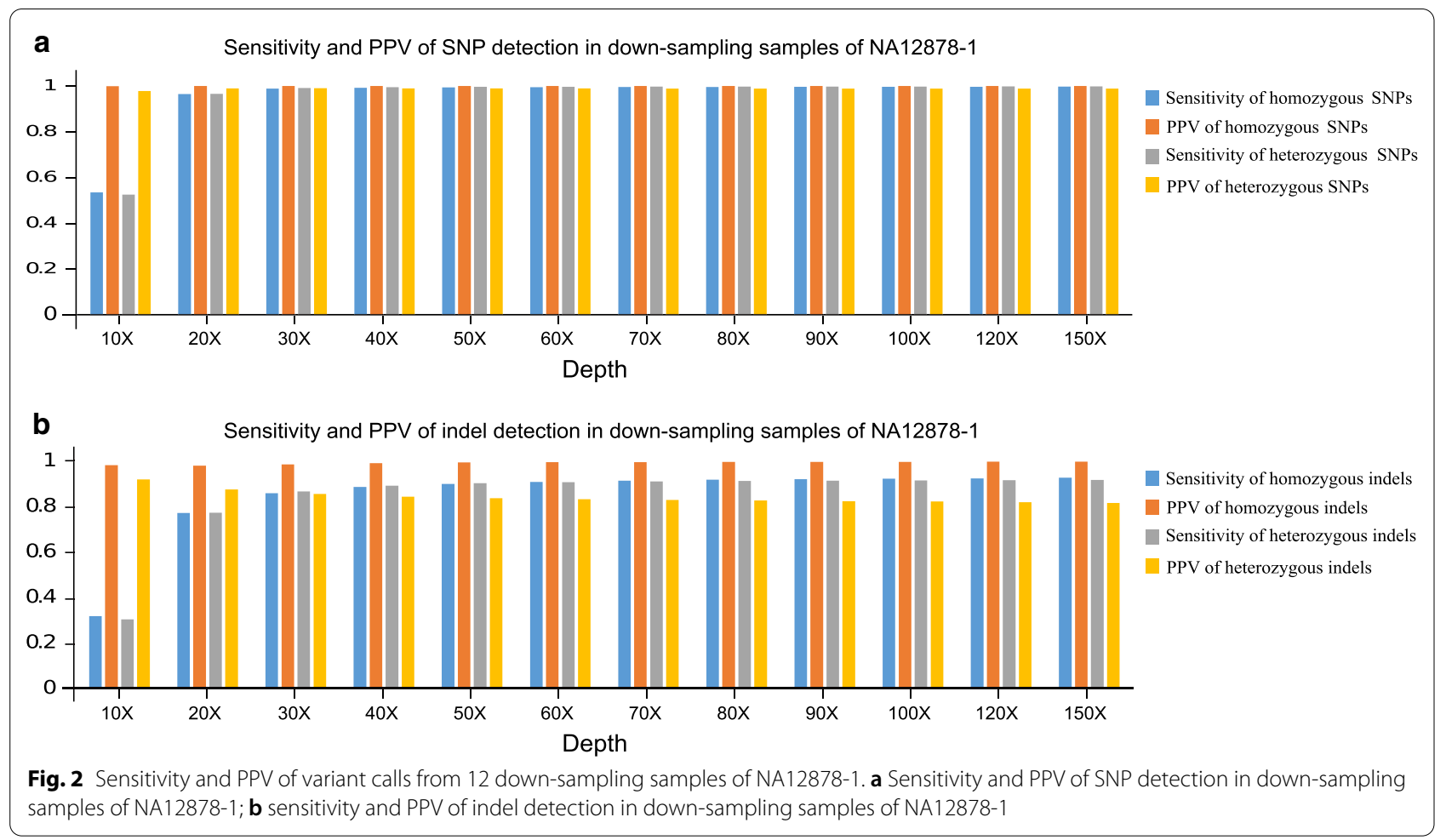

Figure 1 shows the overall design of this study. First, to assess the recommended depth for probandonly WGS in clinical diagnostics, the analysis of the sensitivity and positive predictive value (PPV) of high-confidence SNPs/indels, the sensitivity of CNV detection, and the depth and breadth of coverage for disease-associated genes and CNVs were performed using down-sampling samples of NA12878-1. Downsampling samples were randomly down-sampled to a certain sequencing depth using seqtk (https://github. com/lh3/seqtk). The results of the sensitivity and PPV from a single genome may be difficult to generalize to a range of samples [44]. Consequently, in this part, similar analyses were also performed for downsampling samples of another high depth sequencing sample of $\mathrm{YH}$. After determining the recommended mean DP for the proband-only WGS, the Chinese trios (NA24631, NA24694 and NA24695) with the recommended mean DP were used to test the sensitivity and PPV of trio-based WGS when taking advantage of the family-based trio design in clinical WGS. Using downsampling samples of NA12878-1 and NA12878-2, we also compared the performance of WES and WGS using the recommended mean DP. Finally, we analyzed 8 clinical cases with known disease-causing variants using our WGS pipeline (Fig. 1).

\section{Sensitivity, specificity, accuracy, and positive predictive value of variant calls}

The mean DP was recognized as a general indicator of overall sensitivity for SNV/indel detection [4]. To reveal the sensitivity of proband-only WGS for SNP/indel detection, 12 down-sampling samples of NA12878-1 with an increasing mean DP (10X-150X) were evaluated. For the 12 down-sampling samples of NA12878-1, we restricted the calculation of sensitivity and PPV of SNPs/ indels to the high confidence region (v3.3.2). GQ $(\geq 20)$ and DP $(\geq 10)$ were used to filter out variants with erroneous variant calls. As a result, when the mean depth is more than 40X, the sensitivity of detecting homozygous and heterozygous SNPs is more than $99.25 \%$ and $99.50 \%$, the sensitivity of homozygous and heterozygous indels is more than $88.50 \%$ and $89.09 \%$, respectively. We also restricted the calculation of specificity and accuracy of our method to the high confidence region. Both the overall specificity and accuracy of our method was more than 99.99\% when the mean depth was more than 40X. The PPV (high confidence region) for homozygous and heterozygous SNPs exceeded $99.97 \%$ and $98.96 \%$, and the PPV for homozygous and heterozygous indels exceeded 98.93\% and $84.26 \%$, respectively. Heterozygous indels showed the lowest PPV. Considering the sensitivity of $\mathrm{SNP} /$ indel detection and sequencing costs, a sequencing 
depth of $\sim 40 \mathrm{X}$ provided the best value for $\mathrm{SNP} /$ indel detection, as indicated by the trends in the sensitivity results (Fig. 2).

The sensitivity of homozygous and heterozygous SNPs exceeded $96.48 \%$ and $96.59 \%$, and it reached a plateau even with a mean depth of only $20 \mathrm{X}$. The sensitivity significantly increased with sequencing depth from 10 to $30 \mathrm{X}$ for both homozygous and heterozygous indels, but it reached a plateau at an $\sim 40 \mathrm{X}$ mean depth. A mean depth of $40 \mathrm{X}$ could provide a percentage of more than $99.05 \%$ for sites covering more than $20 \mathrm{X}$ in the high confidence region. However, clinical scientist should know that, even with a DP of $\sim 150 \mathrm{X}$, the sensitivity and PPV is still not $100 \%$. With a DP of $150 \mathrm{X}$, the sensitivity for homozygous and heterozygous SNPs was $99.70 \%$ and $99.81 \%$, and the sensitivity of homozygous and heterozygous indels reached $92.57 \%$ and $91.57 \%$ respectively. With a DP of 150X, the PPV for homozygous and heterozygous SNPs was $99.97 \%$ and $98.82 \%$, and the PPV for homozygous and heterozygous indels reached $99.61 \%$ and $81.42 \%$, respectively.

The results from a single genome may be difficult to generalize to a range of samples [44]. Consequently, in this study, we also performed a sensitivity and PPV analysis of SNPs (in the $1 \mathrm{M}$ validated region) for down-sampling samples of another high-depth sequencing sample of YH (Additional file 2: Table S1). In the $1 \mathrm{M}$ validated region, the alleles were validated by the Illumina $1 \mathrm{M}$ BeadChip [33], and similar results were obtained.

\section{Sensitivity of CNV detection}

To detect the sensitivity of proband-only WGS for CNV detection, the 12 down-sampling samples of NA12878-1 (10X-150X) with increasing mean DP were evaluated. CNVnator (read depth) [28], BreakDancer (read pair) [29] and LUMPY (read depth and read pair) [30] were used for the detection of CNVs for the 12 down-sampling samples. In this study, a total of $3 \mathrm{CNV}$ call datasets were assessed. The overall sensitivity of CNVnator, BreakDancer and LUMPY of the 12 down-sampling samples for CNV call set 1 (BreakDancer only included the detection of deletions in CNV call set 1 ), $\mathrm{CNV}$ call set 2 and $\mathrm{CNV}$ call set 3 is shown in Fig. 3. In general, CNV calling is reliable with an increasing DP (Fig. $3 \mathrm{a}-\mathrm{c}$ ). At increasing sequencing depths, the trends of the sensitivity curves for the $3 \mathrm{CNV}$ tools were different from one another. CNVnator showed a wide range of sensitivity with varying DP, and the sensitivity visibly increased with the mean depth, indicating that the sensitivity of CNV detection was positively correlated with the sequencing depth.

We also observed that the size of the CNVs might influence the sensitivity of CNV tools. The performance of each tool varied along with the size of CNVs (Additional file 1: Figs. S1-S9). Taking the deletions in CNV call set 1 as an example, the widely used tool CNVnator may not be suitable for CNV detection when the size of the CNV is less than $1 \mathrm{~kb}$. When the CNV size was less than $1 \mathrm{~kb}$, the sensitivity significantly increased with sequencing depth

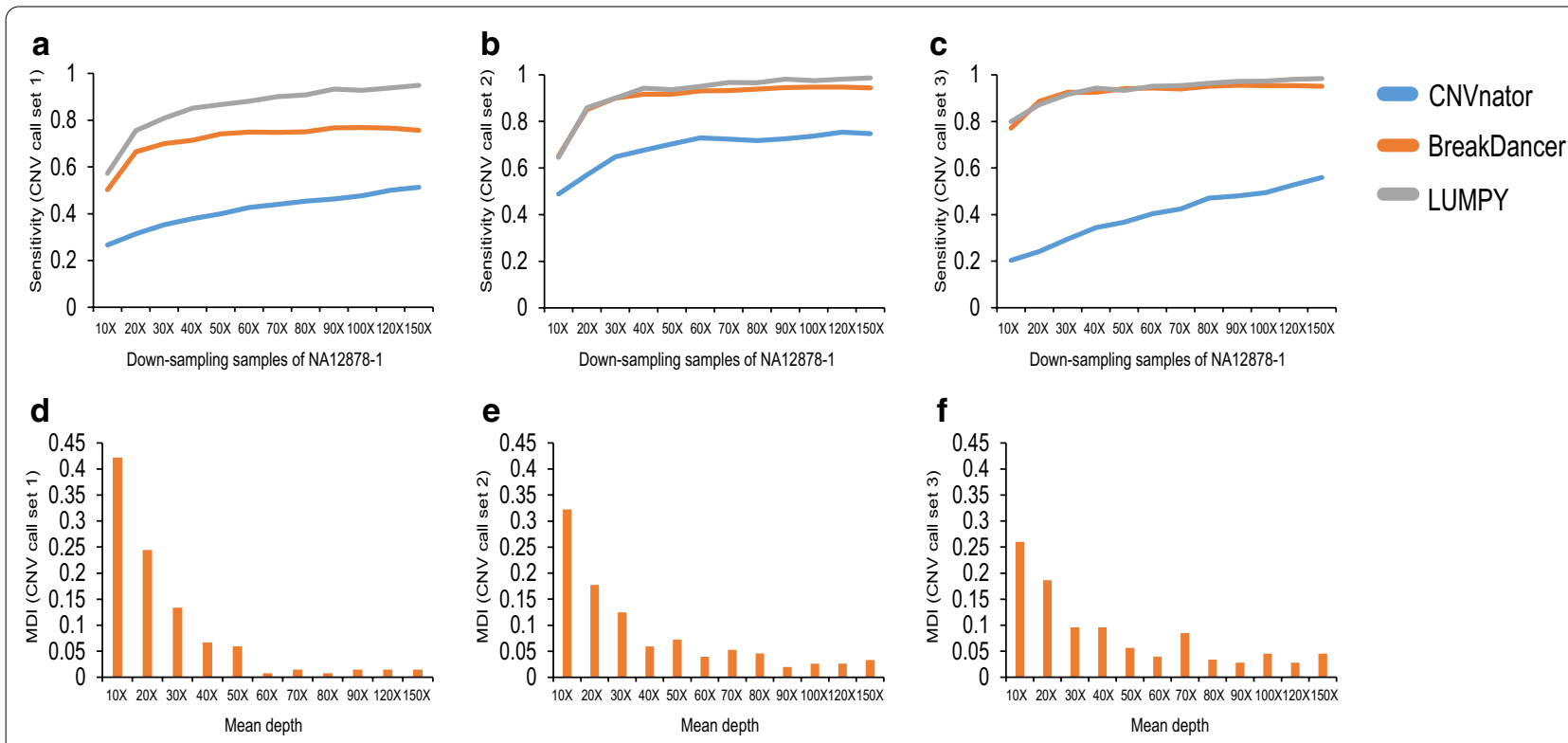

Fig. 3 CNV detection in the 12 down-sampling samples of NA12878-1 using 3 CNV call sets. a Sensitivity of the 12 down-sampling samples of NA12878-1 for CNV call set 1; b sensitivity of the 12 down-sampling samples of NA12878-1 for CNV call set 2; c Sensitivity of the 12 down-sampling samples of NA12878-1 for CNV call set 3; d MDI value for CNV call set 1; e MDI value for CNV call set 2; f MDI value for CNV call set 3 
from 10 to $30 \mathrm{X}$ but reached a plateau at a depth of $\sim 40 \mathrm{X}$ (Additional file 1: Figure S10). This result indicated that when the CNV size was less than $1 \mathrm{~kb}$, the detection rate was greatly influenced by the sequencing depth, which was less obvious when the CNV size ranged between 6 and $70 \mathrm{~kb}$. However, BreakDancer provided a better performance for deletion detection for all CNV sizes. These results suggested that clinical scientists should pay more attention to the selection of $\mathrm{CNV}$ tools when focusing on different CNV sizes.

The selection of CNV call set and CNV detection tools may influence the sensitivity of $\mathrm{CNV}$ detection, making the assessment of the recommended depth for $\mathrm{CNV}$ detection of proband-only WGS difficult. To investigate the minimum requirement of mean DP for $\mathrm{CNV}$ detection in proband-only WGS, using $3 \mathrm{CNV}$ call sets (CNV call set $1,2,3$ ) and the detection results of $3 \mathrm{CNV}$ tools (CNVnator, BreakDancer and LUMPY), we defined a "miss detection index" (MDI) value in this study. The MDI value for a specific mean DP is defined as the frequency when the specific mean DP shows the "lowest" sensitivity for a different $\mathrm{CNV}$ size in a CNV call set. Without regard to selection of the CNV call set and CNV detection tools, MID can be used to evaluate the recommended depth for CNV detection of proband-only WGS.

In the formula, $\mathrm{M}$ indicates the number of times when mean depth i shows the "lowest" sensitivity of CNV detection, and $\mathrm{N}$ indicates the total number of times for all depths showing the "lowest" sensitivity of CNV detection. To obtain qualified CNV sizes in a CNV call set for evaluation, some criteria must be fulfilled for a CNV size (Additional files 1, 2). Detailed criteria and examples of the calculation of MDI can be found in the Additional files $1,2$.

As a result, the MDI value at a depth of 10X, 20X, 30X and 40X ranked first in the down-sampling samples of NA12878-1 (Fig. 3d-f). The 10X-40X accounted for more than $71.98 \%$ of the total depth. Taking together the sensitivity of detecting CNVs and sequencing costs, a sequencing depth of $\sim 40 \mathrm{X}$ provided the best value for $\mathrm{CNV}$ detection, as indicated by the trends in the sensitivity curves (Fig. 3a-c).

\section{Depth and breadth of coverage for disease-associated genes and CNVs}

Although WGS is better than WES for variation detection in patients with genetic disorders, the coverage of coding exons in key disease-associated genes of WGS has not been fully evaluated. To investigate the breadth of coverage of proband-only WGS for disease-associated genes, the breadth of coverage of 6 gene sets for the 12 down-sampling samples of NA12878-1 (10X-150X) and $\mathrm{YH}$ with increasing mean depth were evaluated. For each exon of the coding genes, we calculated the percent of exonic bases covered at more than 10X depth, which was reported to provide $95 \%$ sensitivity for heterozygous SNVs in WES [4]. None of the 12 down-sampling samples of NA12878-1 and YH covered 100\% of the coding exons in the 6 gene sets except for the ACMG59 gene set (Additional file 2: Table S2). The results obtained for the down-sampling samples of NA12878-1 appeared slightly better than down-sampling samples of $\mathrm{YH}$, probably because of the total sequencing depth of NA12878-1 $(\sim 197)$ and YH $(\sim 151)$. Across the 6 gene sets, a limited range of variation was found in the down-sampling samples when the mean depth was more than 40X (Additional file 2: Table S2).

Regarding the ACMG 59 genes, we also observed a range of variation in the breadth of coverage for the 12 down-sampling samples. Thus, a mean depth of more than 70X for $\mathrm{YH}$ and 90X for NA12878-1 covered 100\% for all the ACMG 59 genes. A mean depth of 30X to 50X has been most widely used for WGS $[19,20]$. The proportion of genes in the ACMG59 gene set covering 100\% at $\geq 10 \mathrm{X}$ was $93.22 \%, 98.31 \%$ and $96.61 \%$ for NA128781_30X, NA12878-1_40X and NA12878-1_50X (with mean depths of $\sim 30 \mathrm{X}, \sim 40 \mathrm{X}$ and $\sim 50 \mathrm{X}$ ). For a mean depth of $\sim 30 \mathrm{X}, \sim 40 \mathrm{X}$ and $\sim 50 \mathrm{X}$ for $\mathrm{YH}$, we observed that $86.44 \%, 93.22 \%$ and $98.31 \%$ of the genes covered $100 \%$ at $\geq 10 \mathrm{X}$. The breadths of coverage were significantly better when the average sequencing depth was more than $40 \mathrm{X}$ (Fig. 4a). The sites of all genes covered more than $99.9 \%$ when the sequencing depth was $\sim 40 \mathrm{X}$. Interestingly, we also observed poorly covered RYR1 and TGFBR1 genes in this study (Fig. 4a) in comparison to a previously published paper measuring the sensitivity and coverage of clinical WES [4], indicating that the poor coverage of RYR1 and TGFBR1 might be caused by the features of the gene regions and not the sequencing methods used. Clinical scientists must pay more attention to these genes when performing clinical WGS. Considering the cost of sequencing, a sequencing depth of $\sim 40 \mathrm{X}$ provides the best value for the coverage of the ACMG 59 gene set, as indicated by the trends in breadth of coverage value of the ACMG 59 genes. We observed similar patterns for downsampling samples of YH (Additional file 2: Table S3). We also examined the percentage of genes at $\geq 20 \mathrm{X}$ coverage in the ACMG 59 gene set, which could provide $99 \%$ sensitivity for heterozygous SNVs [4]. We found that $81.36 \%$ and $59.32 \%$ of the genes covered $100 \%$ for NA12878-1 and $\mathrm{YH}$ when the mean DP was $\sim 40 \mathrm{X}$.

CNVs are another major part of human genetic variation, which are often found to be associated with human diseases. For the CNVs in the DECIPHER database, most CNVs could be well covered (more than $95 \%$ coverage) at a depth $>10 \mathrm{X}$ when the sequencing depth was 40X-50X 


\section{a}

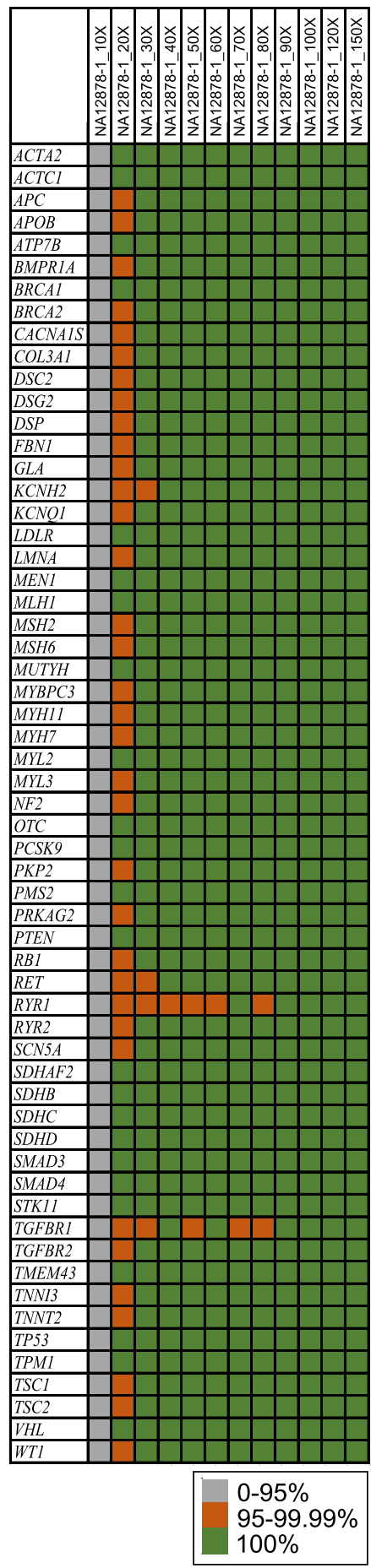

b

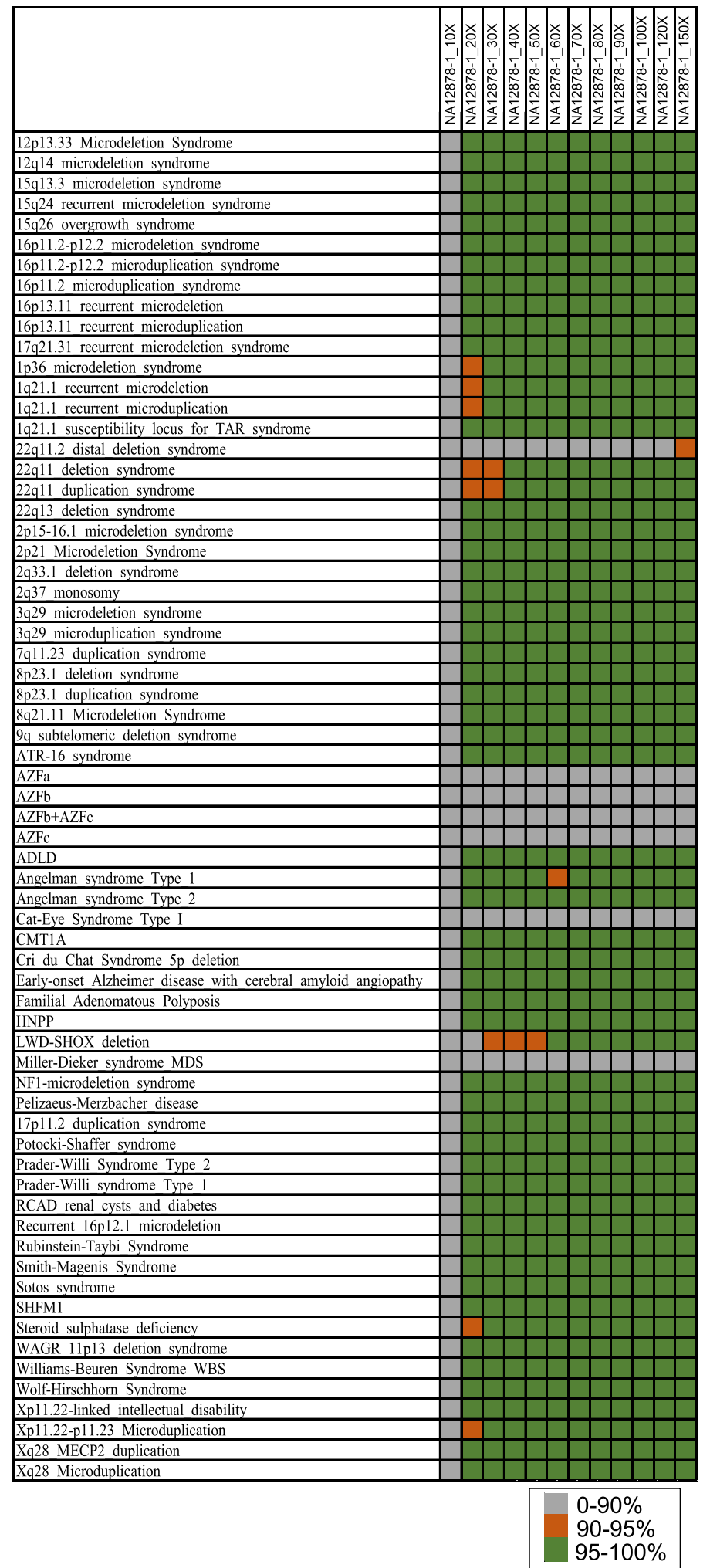

Fig. 4 Depth and breadth of coverage for disease-associated genes and CNVs. a Depth and breadth of coverage for disease-associated genes; $\mathbf{b}$ depth and breadth of coverage for CNVs 
for NA12878-1 (Fig. 4b). Clinical scientists should pay more attention to 22q11.2 distal deletion syndrome, CatEye Syndrome Type I and Miller-Dieker syndrome, which showed a coverage of $88.96 \%, 4.54 \%$ and $84.43 \%$ even with a sequencing depth of 100X. Similar patterns were also observed for down-sampling samples of YH (Additional file 2: Table S4).

\section{Sensitivity and PPV among trios}

The purpose of the analysis of trios was to test the sensitivity and PPV when taking advantage of the family-based trio information in clinical WGS. Although the sequencing depth of the Chinese trio was more than 100, here we used down-sampling samples with a mean depth of 44.00X, 43.77X and 43.05X for NA24631, NA24694 and NA24695, respectively. We concentrated on the depth of $\sim 40 \mathrm{X}$ because the $\sim 40 \mathrm{X}$ depth is the most widely used and recommended depth for WGS, which is also consistent with some of our previous results, especially for $\mathrm{CNV}$ detection.

In this study, we took advantage of the family-based trio design (Fig. 5a) to calculate the sensitivity and PPV in high confidence regions (NISTv3.3.2/GRCh37) [32]. The analysis was restricted to variants with $\mathrm{DP} \geq 10 \mathrm{X}$ and $G Q \geq 20$ (Fig. 5a). We focused on the loci where one parent was homozygous for the alt allele and the other was homozygous for the reference allele. For these variants, the offspring should be heterozygous, thus providing a new "gold standard set" for NA24631 (the offspring). In comparison to the high-confidence calls of NA24631 provided by NIST (the high-confidence call set), the new gold standard set could be used to test the sensitivity and PPV for trio-based WGS. Figure 5b shows the results of the sensitivity and PPV of the "gold standard set" and the

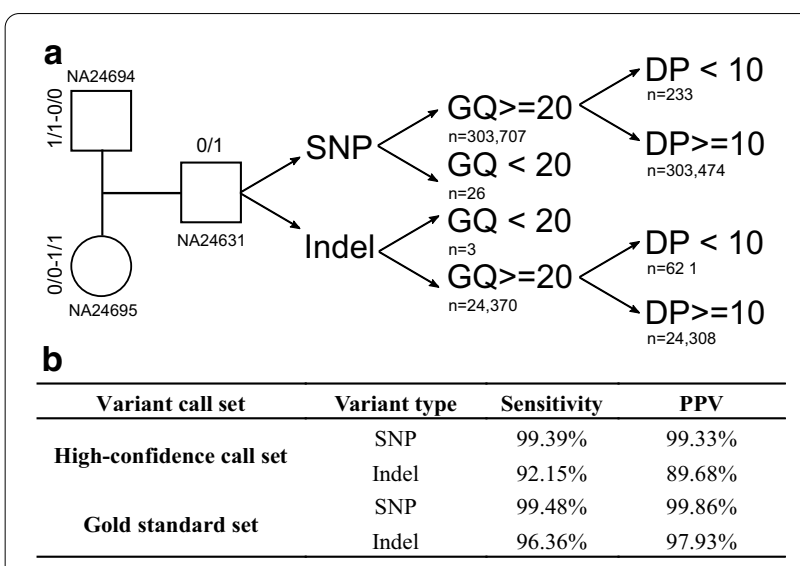

Fig. 5 Variants analysis in the Chinese trios. a Variants used for trios analysis; $\mathbf{b}$ sensitivity and PPV of the "gold standard set" and the high-confidence call set for NA24631 high-confidence call set for NA24631. As a result, the sensitivity of the "gold standard set" for SNP and indel detection was $>99.48 \%$ and $>96.36 \%$, respectively, and the PPVs were $99.86 \%$ and $97.93 \%$. Trio-based analysis showed great improvement for the PPV of indel detection (Fig. 5b), and PPV for indel detection improved from $89.68 \%$ (using the high-confidence call set) to $97.93 \%$ (using the "gold standard set").

\section{WGS and WES}

Here we evaluated the performance of WES (MGIEasy Exome FS Library Prep Set) and WGS (MGIEasy PCRFree DNA Library Prep Set) using DNA samples of NA12878. NA12878-1_40X and NA12878-2_120X for the evaluation. NA12878-1_40X was the down-sampling sample of NA12878-1 with a mean DP of 40X. NA128782_120X was a down-sampling sample of NA12878-2 with a mean DP of 120X, which is typical and the current standard for clinical WES [45]. Two quality parameters for variation detection (DP and GQ), sensitivity for SNV/ indel detection, and the breadth of coverage of the list of 8394 putative disease-associated genes and CNVs were compared in this section.

DP and GQ are two main parameters assessing the quality of variant calls, which are often used to filter out variants with erroneous variant calls [27, 33]. First, we investigated the GQ and DP distribution of NA128781_40X and NA12878-2_120X in the regions of the human genome covered by WES $(59,082,036 \mathrm{bp})$. We found similar results to those obtained in a previous WES study [10]. The distribution of DP for the variants had a wider range in NA12878-2_120X than in NA12878-1_40X (Additional file 1: Figure S11), with a median depth of 94X but a mode at 63X and 58X for SNPs and indels, respectively, indicating low levels of coverage for a substantial proportion of variants. In contrast, the distribution of DP was nearly normal for NA12878-1_40X, with a median at $42 \mathrm{X}$ and coinciding mode at $41 \mathrm{X}$ for both SNPs and indels (Additional file 1: Figure S11). The vast majority of variants called by NA12878-2_120X had a GQ close to 100 and fluctuated along with the GQ scores. The distribution of GQ for variants in NA12878-1_40X showed a mode in low GQ area (with a peak value close to GQ 41), which was probably caused by insufficient variant calls in the WES regions.

For the detection of SNVs and indels, "true positive" calls were further restricted to the regions of the human genome covered by WES $(59,082,036 \mathrm{bp})$. A total of 44,726 variants were used for evaluation. DP and GQ filtering were not used in this part. In general, the sensitivity and PPV of WGS (NA12878-1_40X) were higher than WES (NA12878-2_120X) for the 44,726 variants (Additional file 2: Table S5), except for the PPV 
for homozygous indel detection. For $90.39 \%$ of the gold SNPs, WES and WGS yielded the same genotype. More than $63.41 \%$ of these concordant SNVs were identified as heterozygous, which was similar to those obtained in previous WES studies $[4,20,46]$.

Then, we investigated the breadth of coverage of NA12878-1_40X and NA12878-2_120X in the 8394 putative disease-associated genes and CNVs (DECIPHER database). As a result, WGS showed better coverage of both putative disease-associated genes and CNVs. More than $99.77 \%$ of the exon region sites of the 8394 putative disease-associated genes were covered with a depth $\geq 10$ for NA12878-1_40X, while NA12878-2_120X covered $99.46 \%$ of the exon regions. NA12878-2_120X was poorly covered for CNVs in the DECIPHER database (Additional file 2: Table S6). More than $69.69 \%$ of the CNVs showed a coverage less than $10 \%$.

\section{Analysis of 8 clinical cases with known disease-causing variants}

In this study, samples of 8 clinical cases with known variants of various types were recruited and reanalyzed using our WGS pipeline. All 12 variants (Additional file 2: Table S7) were validated previously by methods other than MPS technology, including 8 SNVs, 3 indels and 1 $\mathrm{CNV}$. Seven and five variants were classified as pathogenic and likely pathogenic, respectively, according to the ACMG guidelines for variant classification [22, 23].

Focusing on SNVs, indels and CNVs, we applied our method to the 8 clinical cases using singleton WGS. Variants were manually assessed for quality and interpreted according to the American College of Medical Genetics and Genomics (ACMG) guidelines for variant classification [22, 23]. All the previously validated variants (Additional file 2: Table S7) were successfully detected using our WGS pipeline, which further demonstrated the sensitivity of the method.

Based on the sequencing DP, the average cost for the WGS approach was $\sim \$ 490$ per sample for the 8 clinical cases in the current study. The overall cost (from DNA extraction to reporting) for a single case with a mean sequencing depth of $\sim 40 \mathrm{X}$ was approximately $\$ 600$, including $\sim 280$ for chemicals (DNA extraction, library construction and sequencing), $\sim \$ 220$ for labor, and $\sim \$ 100$ for depreciation expenses.

\section{Discussion}

Thus far, more than 8000 Mendelian diseases have been recorded by OMIM (Online Mendelian Inheritance in Man), more than 5000 of which have a phenotype description and molecular basis. The rapid development of massively parallel sequencing (MPS) technology has revolutionized the field of genetic diagnosis in the clinical setting, making it possible for MPS to be a routine part of clinical care. The emergence of MPS technology makes multigene sequencing, exome level sequencing, and even genome level sequencing possible, which have been increasingly widely used in clinical diagnoses for genetic diseases. In this study, we performed a comprehensive analysis of the sensitivity and coverage of clinical WGS as a diagnostic test for genetic disorders. First, we analyzed the sensitivity and PPV value of high-confidence SNPs/indels, as well as the sensitivity of CNV detection, using down-sampled NA12878 and YH. A new MDI value was defined for the evaluation of $\mathrm{CNV}$ detection; then, we investigated the depth and breadth of coverage for disease-associated genes and CNVs in downsampling samples of NA12878 and $\mathrm{YH}$. A new gene set and a CNV call set were generated during this process. Next, we compared the performance of WES and WGS for DNA samples of NA12878. We also tested the sensitivity and PPV of variant calls when taking advantage of the family-based trio design in clinical WGS. Finally, we analyzed 8 clinical cases with known disease-causing variants using our WGS pipeline. The results suggested that WGS can be used in the detection of SNVs/indels/ CNVs with high sensitivity, and the current standard of a mean depth of $\sim 40 \mathrm{X}$ may be a cost-effective sequencing depth for SNV/indel detection and the identification of most CNVs. WGS is likely to be widely used and become a routine part of clinical care in the near future.

Although a mean depth of 40X was recommended for clinical WGS in this study, certain disease-causing variants may be detected with low depth (Additional file 2: Table S7, 3 out of 12 variants with 17X). In real clinical setting, these variants will be caught and should be further validated using methods other than MPS. Using the 12 down-sampling samples of NA12878-1, we found that a mean depth of $40 \mathrm{X}$ could provide a percentage of more than $99.05 \%$ for sites covering more than $20 \mathrm{X}$ in the high confidence region, which means that, even reaching a mean depth of $40 \mathrm{X}$, there are still sites with a depth of less than 20X. Variants called at these sites with low depth may be false positives, however, they will still be caught. Further interpretation of these variants should be conducted combined with the phenotype of the patient. If disease related or known diseasing causing mutations are found with low depth, further validation is recommended to be conducted using methods other than MPS.

During the analysis of the PPV of indels in the high confidence regions, we detected a slight decline for heterozygous indels in the 12 down-sampling samples of NA12878 with the increase in DP. One reasonable explanation is that with the increase in depth, both true positives and false positives increased. We found, however, that the false positives increased a little bit faster than the 
true positives, and as a result, PPV declined. A massive scale $\mathrm{p}$ population-based polymorphism database and further filtering of the variants may be useful to solve this problem.

In addition to SNVs and indels, another major part of human genetic variation is copy number variation. According to clinical requirements, choosing suitable methods and tools for accurate and reliable detection of CNVs is important for clinical diagnostics. WGS can detect nearly all known genetic variations. However, our results indicated that, although $\mathrm{CNV}$ calling was reliable with increasing DP, the performance of $\mathrm{CNV}$ tools varied immensely. Finding the right tool for $\mathrm{CNV}$ detection is difficult for clinical scientists. Our results suggested that read pair methods (BreakDancer in particular) showed the best performance for the identification of deletions of more than $1 \mathrm{~kb}$. Moreover, although some "gold standard" CNV call set has been widely used in published papers [40-42], with the lack of validation of various methods, some CNVs may be false positives with inaccurate or low-resolution boundaries. Factors (such as CNV size and the selected "gold standard" set) may also influence the sensitivity of CNV detection, making it difficult to determine the sufficient DP for CNV detection. In this study, we introduced the concept of MDI to solve this problem. The MDI value for a specific mean DP is defined as the frequency when the specific mean DP shows the "lowest" sensitivity for different CNV size in a CNV call set, which was defined to evaluate the recommended depth for CNV detection. Finally, we found that the current standard of a mean depth of $40 \mathrm{X}$ might be sufficient for the identification of most CNVs. Based on the results already obtained, MDI can be used to reflect the performance of $\mathrm{CNV}$ detection for certain $\mathrm{CNV}$ call sets and CNV tools. However, there are limitations of our analysis. First, MDI has not been validated elsewhere. Based on the definition of MDI, we can see that MDI is an objective value for the assessment of recommended depth for CNV detection. Lacking validation may block the application of MDI. Second, we only sampled a small number of CNV callers (CNVnator, BreakDancer and LUMPY). In a real clinical setting, the application of more than one CNV calling algorithm should be considered to improve the sensitivity of CNV detection. Analysis of the sensitivity of CNV detection with all available CNV tools would be an interesting research topic. In addition, we found that additional coverage is associated with an overall increase of the sensitivity for CNV detection; however, this is less obvious as the CNV size is more than $100 \mathrm{~kb}$, as described in another published paper [26].

There is no perfect "gold standard" CNV dataset for benchmarking. Thus, in this study we compiled a list of 2022 "likely true positives" from the 3 most commonly used CNV call sets of NA12878 from published papers [40-42] for benchmarking (Additional file 2: Table S8), including 1912 deletions and 110 duplications. This new set represents a combination of the $3 \mathrm{CNV}$ call sets after evaluation. For this new CNV call set, we defined true positives as the variants detected by at least one CNV tool (CNVnator, BreakDancer and LUMPY) with more than $50 \%$ reciprocal overlap and confirmed by visualization of the copy ratio using an in-house script. This new set is an ideal "gold standard" CNV call set of NA12878 for clinical WGS benchmarking.

When comparing the GQ and DP distribution of NA12878-1_40X (WGS) and NA12878-2_120X (WES), the regions were restricted to the human genome covered by WES $(59,082,036$ bp). In this region, NA128782_120X detected 54,290 SNPs and 7918 indels, while NA12878-1_40X identified 53,107 SNPs and 6577 indels. High depth based WES method (NA12878-2_120X) detected more SNPs and indels than WGS based method (NA12878-1_40X). As is shown in Additional file 1: Figure S11, low levels of coverage for a substantial proportion of variants were detected in NA12878-2_120X. Higher sensitivity for WGS (NA12878-1_40X) were also detected for the 44,726 variants (Additional file 2: Table S5). One reasonable explanation for the different "variation count" in NA12878-1_40X (WGS) and NA12878-2_120X (WES) is that, in the sites with low levels of coverage in NA12878-2_120X, more false positives were called.

The turn-around time and cost of WGS are two key points for the clinical application of WGS. The entire workflow of this method lasts approximately 11-12 days from the recruitment of sample to clinical reporting for one sample. BGI produced MegaBOLT (MegaBOLT bioinformatics analysis accelerator) along with the sequence platform MGISEQ-2000, which is an MGI self-developed and MPS-concentrated hardware accelerating system for bioinformatics analysis. MegaBOLT supports the analysis of WGS and WES, and it is 20 times faster than the traditional GATK approach, which can be used to shorten the bioinformatics process. Along with the development of automated diagnostic tools [47, 48], which could be used to prioritize patient phenotypes and expedite genetic disease diagnosis, the turn-around time of WGS could be further reduced. The overall cost, including chemicals, labor, and depreciation expenses for the WGS approach was $\$ 600$ per sample ( $40 \mathrm{X}$ depth). Sequencing accounted for nearly half of the total cost. In general, variant calling is more reliable with increasing DP. However, there is a detection ceiling for some genes and/or regions (such as regions related to Miller-Dieker syndrome), which cannot be solved by increasing the sequencing 
depth. The cost and sensitivity of WGS must be balanced. Our results suggest that the current standard of a mean depth of 40X may be sufficient for the identification of most SNVs and CNVs. Reduction of the cost and turn-around time would further improve the clinical application of WGS.

\section{Conclusions}

In summary, the successful application of WGS as a diagnostic test for genetic disorders in the real clinical setting requires a comprehensive assessment of the depth and breadth of coverage and the sensitivity of WGS. In this study, we observed variation in the detection of $\mathrm{SNV} /$ indel/CNV and substantial variation in the coverage of medically implicated genes and CNVs. In the real clinical setting, it would be advisable for clinical scientists to determine the range of sensitivity and PPV for different classes of variants for a particular WGS pipeline, which would be useful when interpreting and delivering clinical reports. We believe that WGS is likely to change the clinical diagnosis of rare and undiagnosed diseases in the near future.

\section{Abbreviations}

WGS: Whole-genome sequencing; WES: Whole-exon sequencing; OMIM: Online Mendelian Inheritance in Man; MPS: Massively parallel sequencing; SNV: Single-nucleotide variant; Indels: Small insertions and deletions; CNV: Copy-number variant; ACMG: The American College of Medical Genetics and Genomics; GIAB: Genome in a Bottle; PPV: Positive predictive value; MDI: Miss detection index; DP: Depth of coverage; GQ: Genotype quality; GATK: Genome analysis toolkit.

\section{Supplementary Information}

The online version contains supplementary material available at https://doi. org/10.1186/s12920-021-00948-5.

\section{Additional file 1. Supplementary Tables.}

Additional file 2. Supplementary Material.

\section{Acknowledgements}

We thank all the blood donors for their invaluable contribution to this study.

\section{Authors' contributions}

ZYP, JS, and YS designed the research. YS wrote the first draft of the article. CNF, LJS, XDW, ZYY, ZPX, CNS, HYZ, WD, YL, YQL and JS designed and performed the experiments. FXL, YSW, ZHF, RH, ZHW, JGP and ZYP performed data analysis. HH provided patient specimens and conducted histopathological examinations. ZYP, JS, YS, FXL, CNF, YSW and LS contributed to drafting and revising the manuscript. All authors reviewed the manuscript.

\section{Funding}

This work was supported by the Special Foundation for High-level Talents of Guangdong (Grant 2016TX03R171). This work was also supported by Beijing Municipal Science \& Technology Commission (NO. Z181100001918013). These projects are non-profit research projects by government, and had no role in the study design, data collection and analysis, decision to publish, or preparation of the manuscript.

\section{Availability of data and material}

The datasets of the GIAB samples used and analyzed during the current study have been deposited in the CNSA (https://db.cngb.org/cnsa/) of CNGBdb with accession code CNP0000813. The data of the 8 clinical cases generated and analyzed during the current study is not publicly available as they are patient samples and sharing them could compromise research participant privacy. Other databases used in this study are listed below: GRCh37: http://hgdow nload.soe.ucsc.edu/goldenPath/hg19/chromosomes/. High-confidence calls (SNPs and indels) for GIAB sample HG001 (NA12878) (v3.3.2): ftp://ftp-trace. ncbi.nlm.nih.gov/giab/ftp/release/NA12878_HG001/NISTv3.3.2/GRCh37/. High-confidence calls (SNPs and indels) for GIAB sample HG005 (NA24631) (v3.3.2): ftp://ftp-trace.ncbi.nlm.nih.gov/giab/ftp/release/ChineseTrio/HG005_ NA24631_son/NISTv3.3.2/GRCh37/. High-confidence calls (SNPs and indels) for GIAB sample HG006 (NA24694) (v3.3.2): ftp://ftp-trace.ncbi.nlm.nih.gov/ giab/ftp/release/ChineseTrio/HG006_NA24694_father/NISTv3.3.2/GRCh37/. High-confidence calls (SNPs and indels) for GIAB sample HG007 (NA24695) (v3.3.2): ftp://ftp-trace.ncbi.nlm.nih.gov/giab/ftp/release/ChineseTrio/HG007_ NA24695_mother/NISTv3.3.2/GRCh37/. ClinVar: https://www.ncbi.nlm.nih. gov/clinvar/. Genetic Home Reference: https://medlineplus.gov/genetics/. HGMD: http://www.hgmd.cf.ac.uk/ac/index.php. OMIM: https://omim.org/. Orphanet: https://www.orpha.net/. DECIPHER: https://decipher.sanger.ac.uk/.

\section{Declarations}

\section{Ethics approval and consent to participate}

Written informed consents were obtained from all the participants. This study was approved by THE INSTITUTIONAL REVIEW BOARD OF BGI (NO. BGIIRB19143) and was performed in accordance with the Declaration of Helsinki.

\section{Consent for publication}

Not applicable.

\section{Competing interests}

ZYP is an Editorial Board Member of BMC Medical Genomics. The authors declare that they have no competing interests.

\section{Author details}

${ }^{1}$ BGI Genomics, BGI-Shenzhen, Shenzhen 518083, China. ${ }^{2}$ Tianjin Medical Laboratory, BGI-Tianjin, BGI-Shenzhen, Tianjin 300308, China. ${ }^{3}$ Binhai Genomics Institute, BGI-Tianjin, BGI-Shenzhen, Tianjin 300308, China. ${ }^{4}$ BGI-Beijing Clinical Laboratories, BGI-Shenzhen, Beijing 101300, China.

Received: 20 May 2020 Accepted: 31 March 2021

Published online: 13 April 2021

\section{References}

1. DePristo MA, Banks E, Poplin R, Garimella KV, Maguire JR, Hartl C, et al. A framework for variation discovery and genotyping using next-generation DNA sequencing data. Nat Genet. 2011;43(5):491-8.

2. Meynert AM, Ansari M, FitzPatrick DR, Taylor MS. Variant detection sensitivity and biases in whole genome and exome sequencing. BMC Bioinf. 2014;15:247.

3. Carson AR, Smith EN, Matsui H, Braekkan SK, Jepsen K, Hansen JB, et al. Effective filtering strategies to improve data quality from populationbased whole exome sequencing studies. BMC Bioinf. 2014;15:125.

4. Kong SW, Lee IH, Liu X, Hirschhorn JN, Mandl KD. Measuring coverage and accuracy of whole-exome sequencing in clinical context. Genet Med. 2018;20(12):1617-26.

5. Barbitoff YA, Polev DE, Glotov AS, Serebryakova EA, Shcherbakova IV, Kiselev AM, Kostareva AA, Glotov OS, Predeus AV. Systematic dissection of biases in whole-exome and whole-genome sequencing reveals major determinants of coding sequence coverage. Sci Rep. 2020;10(1):2057.

6. Scala M, Bianchi A, Bisulli F, Coppola A, Elia M, Trivisano M, Pruna D, Pippucci T, Canafoglia L, Lattanzi S, et al. Advances in genetic testing and optimization of clinical management in children and adults with epilepsy. Expert Rev Neurother. 2020;20(3):251-69. 
7. Pang AW, Macdonald JR, Yuen RK, Hayes VM, Scherer SW. Performance of high-throughput sequencing for the discovery of genetic variation across the complete size spectrum. G3 (Bethesda). 2014;4(1):63-5.

8. Fang H, Wu Y, Narzisi G, O'Rawe JA, Barron LT, Rosenbaum J, et al. Reducing INDEL calling errors in whole genome and exome sequencing data. Genome Med. 2014;6(10):89.

9. Meienberg J, Bruggmann R, Oexle K, Matyas G. Clinical sequencing: is WGS the better WES? Hum Genet. 2016;135(3):359-62.

10. Belkadi A, Bolze A, Itan Y, Cobat A, Vincent QB, Antipenko A, et al. Whole-genome sequencing is more powerful than whole-exome sequencing for detecting exome variants. Proc Natl Acad Sci U S A. 2015;112(17):5473-8.

11. McKusick VA. Mendelian Inheritance in Man and its online version. OMIM Am J Hum Genet. 2007;80(4):588-604.

12. Lelieveld SH, Spielmann M, Mundlos S, Veltman JA, Gilissen C. Comparison of exome and genome sequencing technologies for the complete capture of protein-coding regions. Hum Mutat. 2015;36(8):815-22.

13. Lupski JR, Reid JG, Gonzaga-Jauregui C, Rio Deiros D, Chen DC, Nazareth $L$, et al. Whole-genome sequencing in a patient with Charcot-MarieTooth neuropathy. N Engl J Med. 2010;362(13):1181-91.

14. Herdewyn S, Zhao H, Moisse M, Race V, Matthijs G, Reumers J, et al. Whole-genome sequencing reveals a coding non-pathogenic variant tagging a non-coding pathogenic hexanucleotide repeat expansion in C9orf72 as cause of amyotrophic lateral sclerosis. Hum Mol Genet. 2012;21(11):2412-9.

15. Bae BI, Tietjen I, Atabay KD, Evrony GD, Johnson MB, Asare E, et al. Evolutionarily dynamic alternative splicing of GPR56 regulates regional cerebral cortical patterning. Science. 2014;343(6172):764-8.

16. Weedon MN, Cebola I, Patch AM, Flanagan SE, De Franco E, Caswell R, et al. Recessive mutations in a distal PTF1A enhancer cause isolated pancreatic agenesis. Nat Genet. 2014:46(1):61-4.

17. Normand EA, Alaimo JT, Van den Veyver IB. Exome and genome sequencing in reproductive medicine. Fertil Steril. 2018;109(2):213-20.

18. Rehm HL, Bale SJ, Bayrak-Toydemir P, Berg JS, Brown KK, Deignan JL, et al. ACMG clinical laboratory standards for next-generation sequencing. Genet Med. 2013;15(9):733-47.

19. Thiffault I, Farrow E, Zellmer L, Berrios C, Miller N, Gibson M, et al. Clinical genome sequencing in an unbiased pediatric cohort. Genet Med. 2019;21(2):303-10.

20. Stavropoulos DJ, Merico D, Jobling R, Bowdin S, Monfared N, Thiruvahindrapuram $B$, et al. Whole genome sequencing expands diagnostic utility and improves clinical management in pediatric medicine. NPJ Genom Med. 2016;1:1-9.

21. de Vries BB, Pfundt R, Leisink M, Koolen DA, Vissers LE, Janssen IM, et al. Diagnostic genome profiling in mental retardation. Am J Hum Genet. 2005:77(4):606-16.

22. Sharp AJ, Hansen S, Selzer RR, Cheng Z, Regan R, Hurst JA, et al. Discovery of previously unidentified genomic disorders from the duplication architecture of the human genome. Nat Genet. 2006;38(9):1038-42.

23. Girirajan $\mathrm{S}$, Campbell CD, Eichler EE. Human copy number variation and complex genetic disease. Annu Rev Genet. 2011;45:203-26.

24. Zhang $Y$, Haraksingh $R$, Grubert $F$, Abyzov A, Gerstein $M$, Weissman $S$, et al. Child development and structural variation in the human genome. Child Dev. 2013;84(1):34-48.

25. Li H, Durbin R. Fast and accurate short read alignment with BurrowsWheeler transform. Bioinformatics. 2009;25(14):1754-60.

26. Zhou B, Ho SS, Zhang X, Pattni R, Haraksingh RR, Urban AE. Wholegenome sequencing analysis of CNV using low-coverage and paired-end strategies is efficient and outperforms array-based CNV analysis. J Med Genet. 2018:55(11):735-43.

27. McKenna A, Hanna M, Banks E, Sivachenko A, Cibulskis K, Kernytsky A, et al. The Genome Analysis Toolkit: a MapReduce framework for analyzing next-generation DNA sequencing data. Genome Res. 2010;20(9):1297-303.

28. Abyzov A, Urban AE, Snyder M, Gerstein M. CNVnator: an approach to discover, genotype, and characterize typical and atypical CNVs from family and population genome sequencing. Genome Res. 2011;21(6):974-84.
29. Chen K, Wallis JW, McLellan MD, Larson DE, Kalicki JM, Pohl CS, et al. BreakDancer: an algorithm for high-resolution mapping of genomic structural variation. Nat Methods. 2009:6(9):677-81.

30. Layer RM, Chiang C, Quinlan AR, Hall IM. LUMPY: a probabilistic framework for structural variant discovery. Genome Biol. 2014;15(6):R84.

31. Chen S, Zhou Y, Chen Y, Gu J. fastp: an ultra-fast all-in-one FASTQ preprocessor. Bioinformatics. 2018:34(17)::884-90.

32. Zook JM, Chapman B, Wang J, Mittelman D, Hofmann O, Hide W, et al. Integrating human sequence data sets provides a resource of benchmark SNP and indel genotype calls. Nat Biotechnol. 2014;32(3):246-51.

33. Wang J, Wang W, Li R, Li Y, Tian G, Goodman L, et al. The diploid genome sequence of an Asian individual. Nature. 2008;456(7218):60-5.

34. Kalia SS, Adelman K, Bale SJ, Chung WK, Eng C, Evans JP, et al. Recommendations for reporting of secondary findings in clinical exome and genome sequencing, 2016 update (ACMG SF V2.0): a policy statement of the American College of Medical Genetics and Genomics. Genet Med. 2017;19(2):249-55.

35. Landrum MJ, Lee JM, Benson M, Brown G, Chao C, Chitipiralla S, et al. ClinVar: public archive of interpretations of clinically relevant variants. Nucleic Acids Res. 2016:44(D1):D862-8.

36. Mitchell JA, McCray AT. The Genetics Home Reference: a new NLM consumer health resource. AMIA Annu Symp Proc. 2003:936.

37. Cooper DN, Krawczak M. Human gene mutation database. Hum Genet. 1996:98(5):629.

38. Hamosh A, Scott AF, Amberger JS, Bocchini CA, McKusick VA. Online Mendelian Inheritance in Man (OMIM), a knowledgebase of human genes and genetic disorders. Nucleic Acids Res. 2005;33(Database issue):D514-7.

39. Pavan S, Rommel K, Mateo Marquina ME, Hohn S, Lanneau V, Rath A. Clinical Practice Guidelines for Rare Diseases: The Orphanet Database. PLOS ONE. 2017:12(1):e0170365.

40. Haraksingh RR, Abyzov A, Urban AE. Comprehensive performance comparison of high-resolution array platforms for genome-wide Copy Number Variation (CNV) analysis in humans. BMC Genomics. 2017;18(1):321.

41. Parikh $H$, Mohiyuddin $M$, Lam HY, lyer $H$, Chen $D$, Pratt $M$, et al. svclassify: a method to establish benchmark structural variant calls. BMC Genomics. 2016;17:64

42. Pendleton M, Sebra R, Pang AW, Ummat A, Franzen O, Rausch T, et al. Assembly and diploid architecture of an individual human genome via single-molecule technologies. Nat Methods. 2015;12(8):780-6.

43. Quinlan AR, Hall IM. BEDTools: a flexible suite of utilities for comparing genomic features. Bioinformatics. 2010;26(6):841-2.

44. Weisenfeld NI, Yin S, Sharpe T, Lau B, Hegarty R, Holmes L, et al. Comprehensive variation discovery in single human genomes. Nat Genet. 2014;46(12):1350-5

45. Jamal SM, Yu JH, Chong JX, Dent KM, Conta JH, Tabor HK, et al. Practices and policies of clinical exome sequencing providers: analysis and implications. Am J Med Genet A. 2013;161 A(5):935-50.

46. Zhu Q, Hu Q, Shepherd L, Wang J, Wei L, Morrison CD, et al. The impact of DNA input amount and DNA source on the performance of wholeexome sequencing in cancer epidemiology. Cancer Epidemiol Biomarkers Prev. 2015;24(8):1207-13.

47. Robinson PN, Kohler S, Oellrich A, Sanger Mouse Genetics P, Wang $\mathrm{K}$, Mungall CJ, et al. Improved exome prioritization of disease genes through cross-species phenotype comparison. Genome Res. 2014;24(2):340-8.

48. Kohler S, Schulz MH, Krawitz P, Bauer S, Dolken S, Ott CE, et al. Clinical diagnostics in human genetics with semantic similarity searches in ontologies. Am J Hum Genet. 2009;85(4):457-64.

\section{Publisher's Note}

Springer Nature remains neutral with regard to jurisdictional claims in published maps and institutional affiliations. 